\title{
Completely Prime Modules Over Path Algebras for Dynkin Quiver of Type
}

\section{$A_{n}$ and $D_{n}$}

\author{
Risnawita $^{1, *}$ Irawati $^{2}$, I. Muchtadi-Alamsyah ${ }^{2}$ \\ ${ }^{1}$ Faculty of Teaching and Education, IAIN Bukittinggi, Indonesia. Algebra Research Group, Faculty of Mathematics and Natural \\ Sciences, Institut Teknologi Bandung, Jalan Ganesa 10 Bandung 40132, Indonesia. \\ ${ }^{2}$ Algebra Research Group, Faculty of Mathematics and Natural Sciences, Institut Teknologi Bandung, Jalan Ganesa 10 Bandung \\ 40132, Indonesia. \\ *Corresponding author : Email: risnawitaa@gmail.com
}

\section{ABSTRACT}

Let $K$ be a field and $Q$ be a quiver (directed graph), and let $A=K Q$ be the path algebra that corresponds to $Q$ with coefficients in $K$. An $A$-module $M$ is a completely prime (c-prime) module in the sense that $r m=0$ for one $m \in M$ and $r \in A$ implies that either $r$ annihilates all $M$ or $m=0$. In this paper, we prove that for Dynkin quiver type $A_{-} n$ and $D_{-} n$, an $A$-module $M$ is $c$-prime if and only if it is simple.

.Keywords: Completely Prime Module, Path Algebra, Dynkin.

\section{INTRODUCTION}

The concept of a prime module was proposed as a generalization of the prime ideal structure of a ring [2]. Suppose that $M$ is a left module over the ring $R$ (written $R$ - module). A proper submodule $N$ of $M$ is said to be prime if $r R m=0$ with $r \in R$, and $m \in M$ implies $m \in$ $N$ or $r \subseteq M N$ [2]. Various different contexts and example have been studied by [9][15][12]. Irawati ([5],[6]) generalized the concept of the hereditary Noether prime ring (HNP) into the concept of the HNP module. In addition, Wardhana [14] and Saleh [11] discussed the characterization of prime submodules. A module $M$ is said to be a completely prime (c-prime) module over $R$ if $r m=0$ with $r \in R$ and $m \in M$ implies $r M=0$ or $m=0$. We prove that if $M$ is a $c$ prime module, then $M$ is a prime module. If $R$ is a commutative ring, then the prime module is the same as the $c$-prime module. The aim of this study was to explore the notion of c-prime modules in the setting of path algebras.

The path algebra is part of representation theory. The representation theory is used to represent a complex mathematical object into a simpler one. A path algebra
$K Q$ is an algebra over a field $K$ whose basis is the set of all paths in a quiver $Q$. A quiver $Q=\left(Q^{0}, Q^{1}, r, s\right)$ consists of two sets $Q^{0}$ (whose elements are called vertices), $Q^{1}$ (whose elements are called arrows), and two maps , $s, Q^{1} \rightarrow Q^{0}$, which associate to each arrow $e \in Q^{1}$, its source $s(e) \in Q^{0}$, and its range $r(e) \in Q^{0}$, respectively. An algebra can be represented in the form of a quiver and a module can be represented as a quiver representation [1]. The path algebra has been widely studied in ([10], [8], [13], [3]).

In Section 2 of this paper, we will give the basic definitions and notation that will be used in this paper. In Section 3, it is shown that indecomposable simple modules over the path algebra of Dynkin quiver type $A_{n}$ and $D_{n}$ are $c$-prime modules.

\section{PATH ALGEBRA, REPRESENTATION AND MODULE}

This section explains the basic theory of path algebra. We refer to [1] for more details.

A quiver $Q=\left(Q^{0}, Q^{1}, r, s\right)$ consists of two sets $Q^{0}, Q^{1} \quad$ and two maps $s: Q^{1} \rightarrow Q^{0}$ and 
$r: Q^{1} \rightarrow Q^{0}$. The elements of $Q^{0}$ are called vertices and the elements of $Q^{1}$ are called arrows. An arrow $\alpha$ in $Q^{1}$ of source $a=s(\alpha)$ and range $b=r(\alpha)$ is usually denoted by $\alpha: a \rightarrow b$. A quiver $Q=\left(Q^{0}, Q^{1}, r, s\right)$ is usually denoted by $Q=\left(Q^{0}, Q^{1}\right)$ or even simply by $Q$. A quiver $Q$ is said to be finite if $Q^{0}$ and $Q^{1}$ are finite sets. If $s^{-1}(v)$ is a finite set for every $v \in Q^{0}$, then $Q$ is called row finite. A vertex $v$ which emits no arrows is called a sink.

A path in quiver $Q$ is a sequence of arrows $p=e_{1} e_{2} \ldots e_{n}$ such that $r\left(e_{i}\right)=s\left(e_{i}+1\right)$ for all $i$. A finite path in quiver $Q$ is a finite sequence of arrows $p=e_{1} e_{2} \ldots e_{n}$ where $r\left(e_{i}\right)=s\left(e_{i}+1\right)$ for all $i$. In this case, the path $p$ is said to have length $n$, denoted by $l(p)=n$. If $p$ is a path such that $v=s(p)=$ $r(p)$, then $p$ is called a closed path based at $v$. If $r(p)=s(p)$ and $s\left(e_{i}\right) \neq s\left(e_{j}\right)$ for every $i \neq j$, then $p$ is called a cycle. A quiver without cycles is called acyclic.

A path algebra $A=K Q$ is an algebra over a field $K$ whose basis is the set of all paths in quiver $Q$. Multiplication of two paths is given by concatenation if this is defined, and 0 otherwise. Extending this bilinearly one gets an algebra structure. Note that $A$ has a unit if and only if $Q$ has only a finitely many vertices. Let $Q$ be a finite connected quiver. A two-sided ideal of $A$ built by arrows in $Q$ is called an arrow ideal of $A$ and is denoted by $R_{Q}$. A two-sided ideal $I$ of $K Q$ is said to be admissible if there exist $m \geq 2$ such that $R_{Q}^{m} \subseteq$ $I \subseteq R_{Q}^{2}$. If $I$ is an admissible ideal of $A,(Q, I)$ is said to be a bound quiver. The quotient algebra $A / I$ is called a bound quiver algebra. If $A$ is isomorphic to a bound quiver $(Q, I)$, we visualize any (finite dimensional) A-module $M$ as a $K$-linear representation of $(Q, I)$, that is, a family of (finite dimensional) $K$ vector spaces $M_{a}$, with $a \in Q^{0}$ connected by $K$-linear maps $\varphi \alpha: M_{a} \rightarrow M_{b}$ corresponding to arrows $\alpha$ : $a \rightarrow b$ in $Q$, and satisfying some relations induced by I.

Let $Q$ be a finite quiver. A representation $M$ of $Q$ is defined as follows: each vertex $a \in Q^{0}$ associates to a $K$-vector space $M_{a}$ and each arrow $\alpha: a \rightarrow b$ in $Q^{1}$ associates to a $K$-linear map $\varphi_{\alpha}: M_{a} \rightarrow M_{b}$. Such a representation is denoted as $=\left(M_{a}, \varphi_{\alpha}\right)_{a \in Q^{0}, a \in Q^{1}}$, or simply as $M=\left(M_{a}, \varphi_{\alpha}\right)$. It is called a finite dimensional representation if each vector space $M_{a}$ is finite dimensional.

Let $M=\left(M_{a}, \varphi_{\alpha}\right)$ and $M^{\prime}=\left(M^{\prime}{ }_{a}, \varphi^{\prime}{ }_{\alpha}\right)$ be two representations of $Q$. A morphism (of representations) $f: M \rightarrow M^{\prime}$ is a family $f=\left(f_{a}\right)_{a \in Q^{0}}$ of $K$ - linear maps $\left(f_{a}: M_{a} \rightarrow M_{a}^{\prime}\right)_{a \in Q^{0}}$ that are compatible with the structure maps $\varphi \alpha$, that is, for each arrow $\alpha: a \rightarrow b$, we have $\varphi_{\alpha}^{\prime} f_{a}=f_{b} \varphi_{a}$ or, equivalently, the following square is commutative:

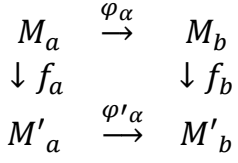

Let $f: M \rightarrow M^{\prime}$ and $g: M^{\prime} \rightarrow M^{\prime \prime}$ be two morphisms of representations of $Q$, where $f=\left(f_{a}\right)_{a \in Q^{0}}$ and $g=$ $\left(g_{a}\right)_{a \in Q^{0}}$. Their composition is defined to be the family $=\left(g_{a} f_{a}\right)_{a \in Q^{0}}$, then $g f$ is easily seen to be a morphism from $M$ to $M^{\prime \prime}$.

The following theorem explains that the category $\bmod A$ whose objects are finitely generated $A$-modules and whose morphisms are $A$-linear maps is equivalent to the category $\operatorname{rep}_{K}(Q, I)$ whose objects are $K$-linear representations of $Q$ that are bounded by $I$ and finite dimensional.

\section{Theorem 2.1. (see [1, Theorem 1.6])}

Let $A=K Q / I$ be connected algebra with finite quiver $Q$ and $I$ be an admissible ideal of $K Q$. There exists a $K$-linear equivalence of categories

$$
F: \bmod A \stackrel{\sim}{\stackrel{\sim}{\rightarrow}} \operatorname{rep}_{K}(Q, I) .
$$

By using Theorem 2.1, we can give an interpretation of a simple, projective and injective module as bound representation. Let $a \in Q^{0}$ define the representation $\left(S(a)_{b}, \varphi_{\alpha}\right)$ of $Q$, denoted by $S(a)$, as follows:

$$
\begin{gathered}
S(a)_{b}= \begin{cases}0 & \text { if } b \neq a \\
K & \text { if } b=a\end{cases} \\
\varphi_{\alpha}=0 \text { for each } \alpha \in Q_{1}
\end{gathered}
$$

Clearly, $S(a)$ is a bound representation of $(Q, I)$. Let $(Q, I)$ be a bound quiver, $A=K Q / I$ and $P(a)=$ $e_{a} A$ where $a \in Q^{0}$. If $P(a)=\left(P(a)_{b}, \varphi_{\beta}\right)$, then $P(a)_{b}$ is the $K$-vector space with as basis the set of all $\bar{\omega}=\omega+I$, with $\omega$ is a path from $a$ to $b$ and for an arrow $\beta: b \rightarrow c$, the $K$-linear map $\varphi_{\beta}: P(a)_{b} \rightarrow$ $P(a)_{c}$ is given by right multiplication by $\bar{\beta}=\beta+I$. If $I(a)=\left(I(a)_{b}, \varphi_{\beta}\right)$, then $I(a)_{b}$ is the dual of $K$ vector space with as basis the set of all $\bar{\omega}=\omega+I$, with $\omega$ is a path from $\mathrm{b}$ to a and for an arrow $\beta: b \rightarrow$ $c$, the $K$-linear map $\varphi_{\beta}: I(a)_{b} \rightarrow I(a)_{c}$ is given by the dual of the left multiplication $\bar{\beta}=\beta+I$.

\section{INDECOMPOSABLE $C$-PRIME MODULES}

This section discusses c-prime modules over the path algebra of a Dynkin quiver of type $A_{n}$ and $D_{n}$.

Definition 3.1. Let $M$ be a left $R$-module. We say that $M$ is a $c$-prime module if for all $r \in R, m \in M$ with $r m=0$, then $r \in \operatorname{Ann}(M)$ or $m=0$.

Dauns gave a general definition of prime modules[2]. 
Definition 3.2. Let $M$ be a left $R$-module, then $M$ is a prime module if for all $r \in R, m \in M$ with $r R m=0$, then $r M=0$ or $m=0$.

If $R$ is a commutative ring, then Definition 3.1 is equivalent to Definition 3.2.

Proposition 3.3. If $M$ is a $c$-prime module, then $M$ is a prime module.

Proof. Suppose $M$ is a $c$-prime module. Let $r \in R$, $m \in M$, with $r R m=0$, then it is clear that $r m=0$. So $r M=0$ and $M$ is a prime module.

The aim of this section is to show that for Dynkin quiver type $A_{n}$ and $D_{n}$, an indecomposable $A$-module $M$ is c-prime if and only if it is simple.

Proposition 3.4. Let $K$ be a field and $A=K A_{n}$ where $A_{n}$ is the quiver in Figure 1 , with $n \geq 1$. Let $M$ be an indecomposable $A$-module, then $M$ is $c$-prime if and only if $M$ is simple.

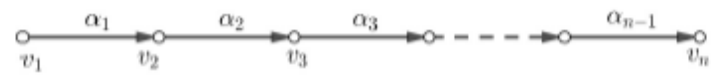

Figure 1. Dynkin Quiver Type $A_{n}$

Proof. In the first step we see the subquiver of Figure 1 in 3 cases.

(i)

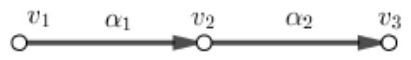

Figure 2. Subquiver $A_{3}$

Let $A_{3}$ be the quiver in Figure 2. Let $M=I\left(v_{1}\right)=$ $S\left(v_{1}\right)$. We will show that $I\left(v_{1}\right)=S\left(v_{1}\right)$ is a $c$-prime module. Let $r \in K A_{3}, m=\left(m_{1}, 0,0\right), m \in M$ with $r m=0$. If $r=v_{1}$, then $m_{1}=0$. If $r=v_{i}, i \neq 1$, then $v_{i} \in$ Ann $M$. If $r=\alpha_{1}$, then $\alpha_{1} \in$ Ann $M$. If $r=\alpha_{2}$, then $\alpha_{2} \in A n n M$. If $r=\alpha_{1} \alpha_{2}$, then $\alpha_{1} \alpha_{2} \in A n n M$ . So $I\left(v_{1}\right)=S\left(v_{1}\right)$ is a $c$-prime module.

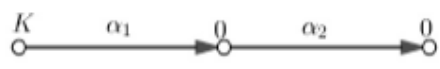

Figure 3. Representation of $I\left(v_{1}\right)=S\left(v_{1}\right)$

Let $A_{3}$ be the quiver in Figure 2. We will show that $M=S\left(v_{2}\right)$ is a $c$-prime module.

Let $r \in K A_{3}, \quad m=\left(0, m_{1}, 0\right), m \in M$ with $r m=0$. If $r=v_{2}$, then $m_{2}=0$. If $r=v_{1} i \neq 2$, then $v_{i} \in$ Ann $M$. If $r=\alpha_{1}$, then $\alpha_{1} \in$ Ann $M$. If $r=\alpha_{2}$, then $\alpha_{2} \in$ Ann $M$. If $r=\alpha_{1} \alpha_{2}$, then $\alpha_{1} \alpha_{2} \in A n n$. So $S\left(v_{2}\right)$ is a $c$-prime module.

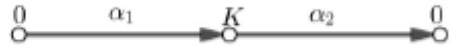

Figure 4. Representation of $S\left(v_{2}\right)$

Let $A_{3}$ be the quiver in Figure 2 .

We will show that $M=S\left(v_{3}\right)=P\left(v_{3}\right)$ is a $c$ prime module.

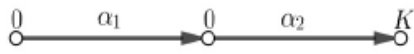

Figure 5. Representation of $S\left(v_{3}\right)=P\left(v_{3}\right)$

Let $r \in K A_{3}, m=\left(0,0, m_{1}\right), m \in M$ with $r m=$ 0 . If $r=v_{3}$, then $m_{1}=0$. If $r=v_{1} i \neq 3$, then $v_{1} \in$ Ann $M$. If $r=\alpha_{1}$, then $\alpha_{1} \in A n n M$. If $r=\alpha_{2}$, then $\alpha_{2} \in A n n M$. If $r=\alpha_{1} \alpha_{2}$ then $\alpha_{1} \alpha_{2} \in A n n M$. So $S\left(v_{3}\right)=P\left(v_{3}\right)$ is a $c$-prime module

Let $A_{3}$ be the quiver in Figure 2. We will show that $M=I\left(v_{2}\right)$ is not a $c$-prime module.

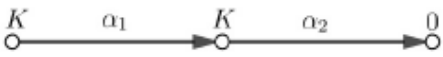

Figure 6. Representation of $I\left(v_{2}\right)$

Let $m=\left(m_{1}, m_{2}, 0\right), m \in M$ and $r=\alpha_{1}$, then $r m=0$. But $\alpha_{1} \notin A n n M$, so $I\left(v_{2}\right)$ is not a $c$-prime module.

Let $A_{3}$ be the quiver in Figure 2. We will show that $M=P\left(v_{2}\right)$ is not a $c$-prime module.

Let $m=\left(0, m_{2}, m_{3}\right), m \in M$ and $r=\alpha_{2}$, then $r m=0$. But $\alpha_{2} \notin$ Ann $M$, so $P\left(v_{2}\right)$ is not a $c$-prime module. So by looking at the subquiver in Figure 2, we can see that $M$ is a $c$-prime module if and only if $M$ is simple.

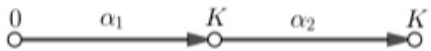

Figure 7. Representation of $P\left(v_{2}\right)$

Let $A_{3}$ be the quiver in Figure 2. We will show that $M=P\left(v_{1}\right)=I\left(v_{3}\right)$ is not a $c$-prime module.

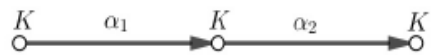

Figure 8. Representation of $P\left(v_{1}\right)=I\left(v_{3}\right)$

Let $m=\left(m_{1}, m_{2}, m_{3}\right), m \in M$ and $r=\alpha_{1}$, then $r m=0$. But $\alpha_{1} \notin A n n M$, so $P\left(v_{1}\right)=I\left(v_{3}\right)$ is not a $c$ prime module.

Thus, by looking at the subquiver in Figure 2, we can see that $M$ is a $c$-prime module if and only if $M$ is simple. 
(ii)

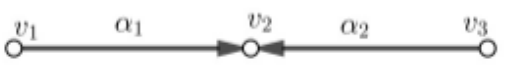

Figure 9. Subquiver $A_{3}(1)$

Let $A_{3}$ be the quiver in Figure 9. Let $M=I\left(v_{1}\right)=$ $S\left(v_{1}\right)$. We will show that $I\left(v_{1}\right)=S\left(v_{1}\right)$ is a $c$-prime module. Let $r \in K A_{3}, m=\left(m_{1}, 0,0\right), m \in M$ with $r m=0$. If $r=v_{1}$, then $m_{1}=0$. If $r=v_{i} i \neq 1$, then $v_{i} \in$ Ann $M$. If $r=\alpha_{1}$, then $\alpha_{1} \in$ Ann $M$. If $r=\alpha_{2}$, then $\alpha_{2} \in A n n M$. So $I\left(v_{1}\right)=S\left(v_{1}\right)$ is a $c$-prime module.

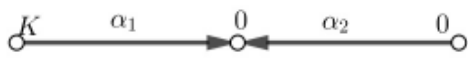

Figure 10. Representation of $I\left(v_{1}\right)=S\left(v_{1}\right)$

Let $A_{3}$ be the quiver in Figure 9. Let $M=P\left(v_{2}\right)=$ $S\left(v_{2}\right)$. We will show that $P\left(v_{2}\right)=S\left(v_{2}\right)$ is a $c$-prime module. Let $r \in K A_{3}, m=\left(0, m_{1}, 0\right), m \in M$ with $r m=0$. If $r=v_{2}$, then $m_{1}=0$. If $r=v_{i}, i \neq 2$, then $v_{i} \in A n n M$. If $r=\alpha_{i}, i \in\{1,2\}$, then $\alpha_{i} \in$ Ann $M$. So $P\left(v_{2}\right)=S\left(v_{2}\right)$ is a $c$-prime module.

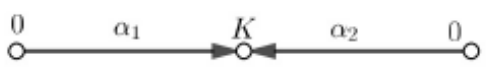

Figure 11. Representation of $P\left(v_{2}\right)=S\left(v_{2}\right)$

Let $A_{3}$ be the quiver in Figure 9. Let $M=I\left(v_{3}\right)=$ $S\left(v_{3}\right)$. We will show that $I\left(v_{3}\right)=S\left(v_{3}\right)$ is a $c$-prime module. Let $r \in K A_{3}, m=\left(0,0, m_{1}\right), m \in M$ with $r m=0$. If $r=v_{3}$, then $m_{1}=0$. If $r=v_{i}, i \neq 3$, then $v_{i} \in A$ Ann $M$. If $r=\alpha_{i}, i \in\{1,2\}$, then $\alpha_{i} \in A n n M$. So $I\left(v_{3}\right)=S\left(v_{3}\right)$ is a $c$-prime module.

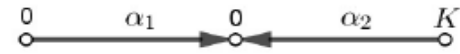

Figure 12. Representation of $I\left(v_{3}\right)=S\left(v_{3}\right)$.

Let $A_{3}$ be the quiver in Figure 9. We will show that $M=P\left(v_{1}\right)$ is not a $c$-prime module.

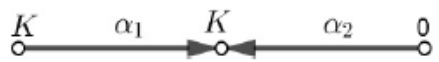

Figure 13. Representation of $P\left(v_{1}\right)$

Let $r \in K A_{3}, m=\left(m_{1}, m_{2}, 0\right), m \in M$ and $r=\alpha_{1}$, then $r m=0$. But $\alpha_{1} \notin A n n M$. So $P\left(v_{1}\right)$ is not a $c$ prime module.

Let $A_{3}$ be the quiver in Figure 9. We will show that $M=P\left(v_{3}\right)$ is not a $c$-prime module.

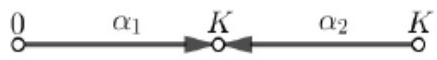

Figure 14. Representation of $P\left(v_{3}\right)$
Let $r \in K A_{3}, m=\left(0, m_{1}, m_{2}\right), m \in M$ and $r=\alpha_{2}$, then $r m=0$. But $\alpha_{2} \notin A n n M$. So $P\left(v_{3}\right)$ is not a $c$ prime module.

Let $A_{3}$ be the quiver in Figure 9. We will show that $M=I\left(v_{2}\right)$ is not a $c$-prime module.

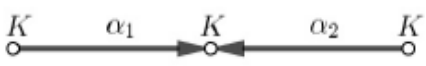

Figure 15. Representation of $I\left(v_{2}\right)$

Let $r \in K A_{3}, m=\left(m_{1}, m_{2}, m_{3}\right), m \in M$ and $r=\alpha_{1}$, then $r m=0$. But $\alpha_{1} \notin A n n M$. So $I\left(v_{2}\right)$ is not a $c$ prime module. So by looking at the subquiver in Figure 9 , we can see that $M$ is a c-prime module if and only if $M$ is simple.

(iii)

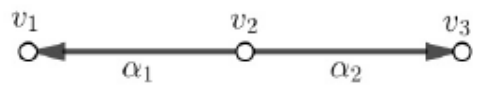

Figure 16. Subquiver $A_{3}(2)$

Let $A_{3}$ be the quiver in Figure 16. Let $M=P\left(v_{1}\right)=$ $S\left(v_{1}\right)$. We will show that $P\left(v_{1}\right)=S\left(v_{1}\right)$ is a $c$-prime module.

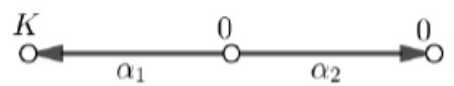

Figure 17. Representation of $P\left(v_{1}\right)=S\left(v_{1}\right)$

Let $r \in K A_{3}, m=\left(m_{1}, 0,0\right), m \in M$ with $r m=0$. If $r=v_{1}$, then $m_{1}=0$. If $r=v_{i}, i \neq 1$, then $v_{i} \in$ Ann $M$. If $r=\alpha_{i}$, then $\alpha_{i} \in A n n M$. If $r=\alpha_{2}$, then $\alpha_{2} \in$ Ann $M$. So $\left(v_{1}\right)=S\left(v_{1}\right)$ is a $c$-prime module.

Let $A_{3}$ be the quiver in Figure 16. We will show that $M=I\left(v_{2}\right)=S\left(v_{2}\right)$ is a $c$-prime module.

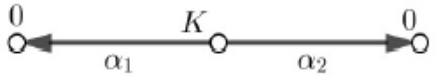

Figure 18. Representation of $I\left(v_{2}\right)=S\left(v_{2}\right)$

Let $r \in K A_{3}, m=\left(0, m_{1}, 0\right), m \in M$ with $r m=0$. If $r=v_{2}$, then $m_{1}=0$. If $r=v_{i} i \neq 2$, then $v_{i} \in$ Ann $M$. If $r=\alpha_{1}$, then $\alpha_{1} \in A n n M$. If $r=\alpha_{2}$, then $\alpha_{2} \in$ Ann $M$. So $I\left(v_{2}\right)=S\left(v_{2}\right)$ is a $c$-prime module.

Let $A_{3}$ be the quiver in Figure 16. We will show that $M=S\left(v_{3}\right)=P\left(v_{3}\right)$ is a $c$-prime module.

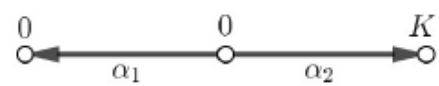

Figure 19. Representation of $S\left(v_{3}\right)=P\left(v_{3}\right)$

Let $r \in K A_{3}, m=\left(0,0, m_{1}\right), m \in M$ with $r m=0$. If $r=v_{3}$, then $m_{1}=0$. If $r=v_{i}, i \neq 3$, then $v_{i} \in$ Ann $M$. If $r=\alpha_{1}$, then $\alpha_{1} \in$ Ann $M$. If $r=\alpha_{2}$, then $\alpha_{2} \in$ Ann $M$. So $S\left(v_{3}\right)=P\left(v_{3}\right)$ is a $c$-prime module. 


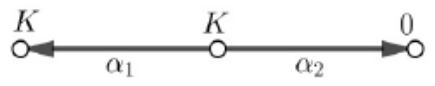

Figure 20. Representation of $I\left(v_{1}\right)$

Let $A_{3}$ be the quiver in Figure 16. We will show that $M=I\left(v_{1}\right)$ is not a c-prime module.

Let $r \in K A_{3}, m=\left(m_{1}, m_{2}, 0\right), m \in M \quad$ and $r=\alpha_{1}$, then $r m=0$. But $\alpha_{1} \notin A n n M$. So $I\left(v_{1}\right)$ is not a cprime module.

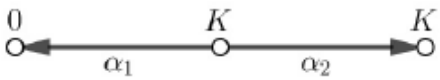

Figure 21. Representation of $I\left(v_{1}\right)$

Let $A_{3}$ be the quiver in Figure 16. We will show that $M=P\left(v_{3}\right)$ is not a $c$-prime module.

Let $r \in K A_{3}, m=\left(0, m_{1}, m_{2}\right), m \in M$ and $r=\alpha_{2}$, then $r m=0$. But $\alpha_{2} \notin A n n M$. So $I\left(v_{3}\right)$ is not a $c$-prime module.

Let $A_{3}$ be the quiver in Figure 16. We will show that $M=P\left(v_{2}\right)$ is not a $c$-prime module.



Figure 22. Representation of $P\left(v_{2}\right)$

Let $m=\left(m_{1}, m_{2}, m_{3}\right), m \in M$ and $r=\alpha_{2}$, then $r m=$ 0 . But $\alpha_{2} \notin A n n M$. So $P\left(v_{2}\right)$ is not a $c$-prime module.

So by looking at the subquiver in Figure 16, we can see that $M$ is a $c$-prime module if and only if $M$ is simple.

Next, we can prove for $v_{i}$ with $i=\{1,2, \ldots, n\}$. Let $A_{n}$ with $n \geq 1$. Let $I\left(v_{1}\right)=S\left(v_{1}\right)$. We will show that $M=I\left(v_{1}\right)=S\left(v_{1}\right)$ is a $c$-prime module. Let $r \in$ $K A_{n}, m=\left(m_{1}, 0, \cdots, 0\right), m \in M$ with $r m=0, r m=$ $r\left(m_{1}, 0, \cdots, 0\right)=(0,0, \cdots, 0)$. If $r=v_{1}$, then $m_{1}=$ 0 . If $r=v_{i}, i \neq 1$, then $v_{i} \in$ Ann $M$. If $r=\alpha_{j}, j \in$ $\{1, \cdots, n-1\}$, then $\alpha_{j} \in$ Ann $M$.

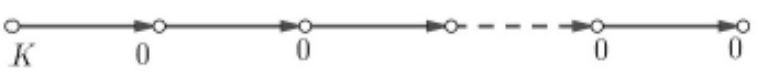

Figure 23. Representation of $S\left(v_{1}\right)$

If $r=\alpha_{j} \alpha_{j+i} \ldots . \alpha_{k}$ with $j, k \in\{1, \cdots, n-1\}, j<k$, then $\alpha_{j} \alpha_{j+i} \ldots \alpha_{k} \in$ Ann $M$. So $I\left(v_{1}\right)=S\left(v_{1}\right)$ is a $c$ prime module.

We will show that $M=S\left(v_{i}\right)$, for $i=1,2, \ldots, n-$ 1 is a c-prime module.

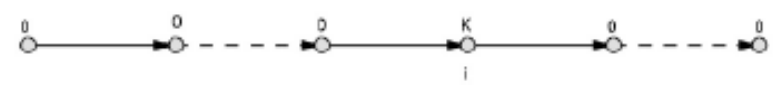

Figure 24. Representation of $S\left(v_{i}\right)$
Let $r \in K A_{n}, m=\left(0,0, \cdots, m_{1}, \cdots, 0\right), m \in M \quad$ with $r m=0, r m=r\left(0,0, \cdots m_{1}, \cdots, 0\right)=(0,0, \cdots, 0)$. If $r=v_{j}, j \neq i$, then $v_{j} \in A n n M$. If $r=v_{i}$, then $m_{i}=$ 0 . If $r=\alpha_{j}$, then $\alpha_{j} \in$ Ann $M$. If $r=\alpha_{j} \alpha_{j+i} \ldots \alpha_{k}$ with $j, k \in\{1, \cdots, n-2\}, j<k, \quad$ then $\alpha_{j} \alpha_{j+i} \ldots \alpha_{k} \in$ Ann $M$. So $S\left(v_{i}\right)$ is a $c$-prime module.

Next, we will show that $M=P\left(v_{n}\right)=S\left(v_{n}\right)$ for $i=1,2, \cdots, n$ is a c-prime module.

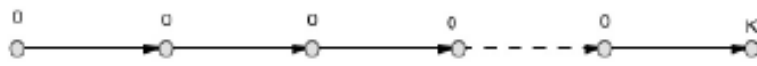

Figure 25. Representation of $S\left(v_{n}\right)$

Let $r \in K A_{n}, m \in M$ with $r m=0, m=(0,0,0, \cdots$ , $\left.m_{n}\right)$. If $r=v_{i}, i \neq n$, then $v_{i} \in$ Ann $M$. If $r=v_{n}$, then $m_{n}=0$. If $r=\alpha_{j}$, then $\alpha_{j} \in A n n M$. If $r=$ $\alpha_{j} \alpha_{j+1} \ldots \alpha_{k}, \quad$ then $\alpha_{j} \alpha_{j+1} \ldots \alpha_{k} \in A n n M$. So $P\left(v_{n}\right)=S\left(v_{n}\right)$ is a $c$-prime module for $i=1,2, n-1$.

Next we will show that $M=I\left(v_{n}\right)=P\left(v_{1}\right)$ is not a c-prime module.

Let $r=\alpha_{1}, m=\left(0, m_{2}, m_{3}, \ldots, m_{n}\right) \neq 0$, then $r m=$ 0 but $\alpha_{1} \notin A n n M$. So $I\left(v_{n}\right)=P\left(v_{1}\right)$ is not a $c$-prime module.

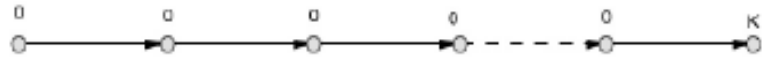

Figure 26. Representation of $I\left(v_{n}\right)$

Next we will show that $M=I\left(v_{i}\right)$ is not a $c$-prime module, for $i=2,3, \cdots, n-1$.

Let $m=\left(m_{1}, m_{2}, \ldots m_{i-1}, 0,0, \ldots, 0\right)$, and $r=\alpha_{i}$, then $r m=0$. But $\alpha_{i} \notin A n n M$. So $I\left(v_{i}\right)$ is not a $c$-prime module.

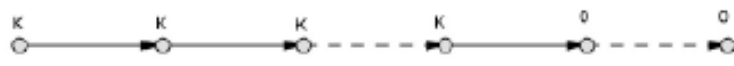

Figure 27. Representation of $I\left(v_{i}\right)$

Next we will show that for another indecomposable module $M$ over path algebra $K A_{n}, M$ is not a $c$-prime module. We can see the representation of $M$ in the Figure 28.

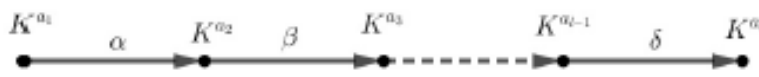

Figure 28. Representation of $M$

Suppose that $r$ in $K A_{n}, \quad m \in M$, where $m=$ $\left(m_{1}, m_{2}, \ldots . m_{l}\right)$ with $r m=0$.

Let $m_{1}=\left(m_{(1,1)}, m_{(2,1)}, \ldots . m_{\left(a_{1,1}\right)}\right)$,

$m_{2}=\left(m_{(2,1)}, m_{(2,2)}, \ldots . m_{\left(a_{2,2}\right)}\right)$, and

$m_{l}=\left(m_{(1, l)}, m_{(2, l)}, \ldots . m_{\left(a_{l}, l\right)}\right)$ 
Without loss of generality, if $r=\alpha$ then $\varphi_{\alpha}\left(m_{1}\right)=0$. Thus $m_{1} \in \operatorname{Ker} \varphi_{\alpha}$. If $\varphi_{\alpha}$ is an injective, then $m_{1}=0$. Suppose that $m=\left(0, m_{2}, \ldots . m_{l}\right) \neq 0$. Therefore $r \notin$ $A n n M$. If $\varphi_{\alpha}$ is not injective, $0 \neq m_{1} \in \operatorname{Ker} \varphi_{\alpha}$. Suppose that $m^{\prime}=\left(m_{1}^{\prime}, m_{2}^{\prime}, \ldots, m_{l}^{\prime}\right)$ with $m_{1}^{\prime} \operatorname{Ker} \varphi_{\alpha}$, then $r m^{\prime} \neq 0$, such that $r \in A n n M$. So $M$ is not a $c$ prime module.

Proposition 3.5. Let $K$ be a field and $A=K D_{n}$, where $D_{n}$ is the quiver in Figure 28, with $n \geq 4$. Let $M$ be an indecomposable $A$-module, then $M$ is $c$-prime if and only if $M$ is simple.

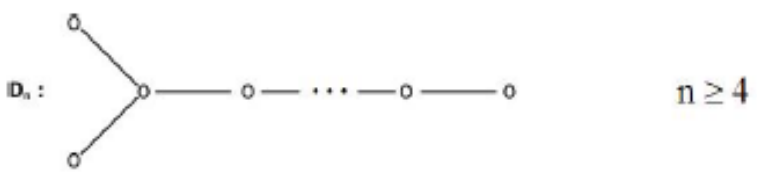

Figure 29. Dynkin Quiver Type $D_{n}$

Proof. The proof is similar as that for Proposition 3.4.

\section{CONCLUSION}

In this paper, we have discussed about $c$-prime modules over path algebra for Dykin quiver type $A_{n}$ and $D_{n}$. The conclusion is as follows:

a. Let $K$ be a field and $A=K A_{n}$ where $A_{n}$ is the quiver in Figure 1, with $n \geq 1$. Let $M$ be an indecomposable A-module, then $M$ is c-prime if and only if $M$ is simple.

b. Let $K$ be a field and $A=K D_{n}$, where $D_{n}$ is the quiver in Figure 29, with $n \geq 4$. Let $M$ be an indecomposable $A$-module, then $M$ is $c$ prime if and only if $M$ is simple.

\section{REFERENCES}

[1] I.Assem, D.Simson, A.Skowron'ski, Elements of the Representation Theory of Associative Algebras 1, London Mathematical Society Student Texts 65, Cambridge University Press, (2006).

[2] J. Dauns, Modules and Rings, Cambridge university press, (1994).

[3] Faisal, I. Muchtadi-Alamsyah, Characterization of Nakayama m-Cluster Tilted Algebra of Type $A_{n}$, accepted in Journal of Indonesian Math. Soc.

[4] D. Kariman, I.M.Maris, Risnawita, Dual Right Serial Algebras, in Advances in Algebraic Structures, Proceeding International Conference in Algebra, World Scientific, 2012.
[5] Irawati, Struktur Modul di Sekitar Modul Dibangun secara Hingga atas Gelanggang HNP dan Generalisasi Gelanggang HNP, Disertasi, Departemen Matematika ITB, (2004).

[6] Irawati, The generalization of HNP ring and finitely generated modules over HNP ring, International Journal of Algebra vol 5 no 13, (2011), 611-626.

[7] N. Jacobson, Structure of Rings vol 37, Amer. Math. Soc, (1956).

[8] D. Kariman, I.M.Maris, Risnawita, Dual Right Serial Algebras, in Advances in Algebraic Structures, Proceeding International Conference in Algebra, World Scientific, (2012).

[9] C Lomp and A. J. Pena, A Note on Prime Modules, Divulgaciones Matematicas vol 8 (2000), 1,31-42.

[10] I.Muchtadi-Alamsyah, Braid action on derived category of Nakayama algebras, Communication in Algebra vol. 36 Issue 07, (2008), 2455-2569.

[11] K. Saleh, P.Astuti, I. Muchtadi-Alamsyah, On the structure of finitely generated primary modules, JP Journal of Algebra, Number Theory and Applications vol 38 Issue 5 (2016), 519 533

[12] N. V. Sanh, An Introduction to some kinds of Radicals in Module Theory, SouthEast Asian conference on Mathematics and its Applications, (2013).

[13] A.P. Santika and I. Muchtadi-Alamsyah, The pregular subspaces of symmetric Nakayama algebras and algebras of dihedral and semidihedral type, JP Journal of Algebra Number Theory and Applications vol 27 No 2 (2012), 131-142.

[14] I.G.A.W.Wardhana, P.Astuti, I. MuchtadiAlamsyah, On almost prime submodules of a module over a principal ideal domain, JP Journal of Algebra, Number Theory and Applications vol 38 Issue 2, (2016), 121-128.

[15] I. E. Wijayanti, Coprime Modules and Comodules. Journal of Algebra, vol 305, number 2,(2006), 937-948. 